\title{
Advanced Hybrid Recording Media
}

\author{
Zuoyi Li, Xiaomin Cheng, Fang Jin, Zhen Li, Gengqi Lin, Xiaofei Yang \\ Department of Electronic Science \& Technology, Huazhong University of Science \& \\ Technology, Wuhan, Hubei, China, 430074
}

\begin{abstract}
Combining the advantages of hard-disk magnetic recording and magneto-optical recording, hybrid recording is regarded as promising candidate for extremely-high density recording technology beyond Tera bits/in ${ }^{2}$. To obtain such high areal density, hybrid recording media are required to have high coercivity and large remanent magnetization at room temperature, the desired temperature dependence of coercivity and magnetization, as well as very short thermal response time. Currently, the researches on the hybrid recording media mainly involve the magnetic hard-disk polycrystalline recording media and the magneto-optical amorphous media. This report presents the latest progresses in the researches on these two kinds of media for hybrid recording.
\end{abstract}

\section{Introduction}

Continuing increase of the areal density is the ultimate motivity to push magnetic data storage research to higher levels. In last decade, accelerated areal density growth has brought researcher's concern to the physic limitation in longitudinal recording caused by the superparamagnetic effect ${ }^{[1,2]}$. This effect means that the continuing reduction of the average grain size required by increasing areal density and signal-to-noise ratio (SNR) consideration leads to the reduction of the stability for thermal magnetization reversal. The thermal stability factor of thin film recording media is given by $K_{u} V / k_{B} T$, where $K_{u}$ is the grain anisotropy, $V$ the switching volume, $\mathrm{k}_{\mathrm{B}}$ the Boltzmann constant and $\mathrm{T}$ the absolute temperature. A minimum stability factor of about 65 is required in longitudinal recording media to avoid thermally driven demagnetization of bit transition and consequent loss of data within the desired storage period of about 10 years ${ }^{[2]}$. To adopt materials with high $\mathrm{K}_{\mathrm{u}}$, therefore, high $\mathrm{H}_{\mathrm{c}}$, is an approach to improve the thermal stability of recording media ${ }^{[3]}$. However, this approach is limited because the values of $\mathrm{H}_{\mathrm{c}}$ must be lower than about half the maximum head write field in order for the head to be able to write the media in both longitudinal and perpendicular recording ${ }^{[4]}$. This dilemma has made a "slowing down" in areal density increases ${ }^{[5]}$. Fig.1 gives the roadmap of the areal density increases of hard-disk drive (HDD) media and magneto-optical (MO) recording media. Obviously, HDD media supports much higher areal density recording now and has 


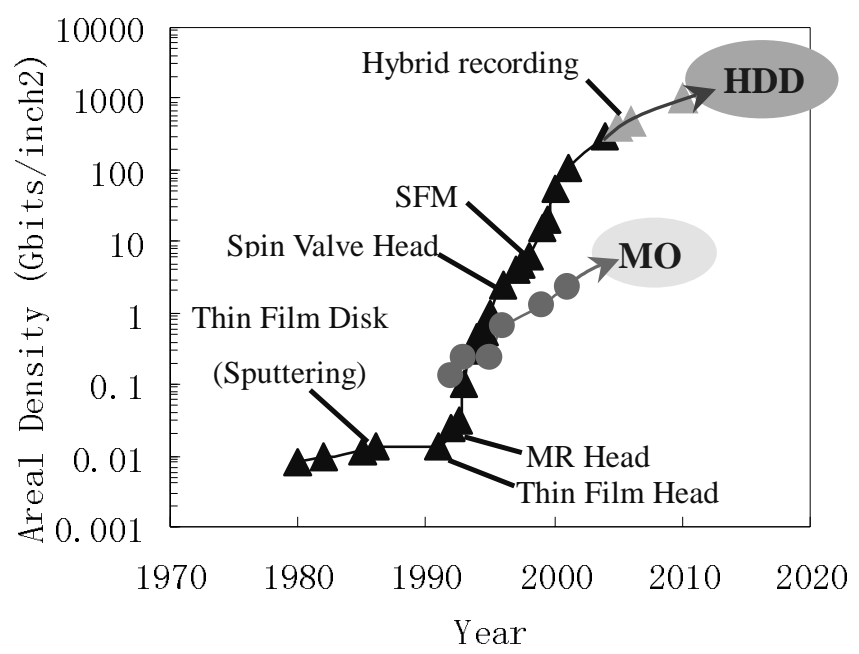

Fig.1 The roadmap of the areal density increases of hard-disk drive (HDD) media and magneto-optical (MO) recording media.

experienced tremendous development in areal density since 1990. The annual growth rate was nearly $100 \%$ in 1990 s and decreased to over $60 \%$ after 2000 . This deceleration is caused by the above-mentioned dilemma.

Hybrid recording, also named thermally assisted magnetic recording (TAMR) or heat assisted magnetic recording (HAMR), gives a way to escape from this dilemma ${ }^{[6,}$ 7]. Hybrid recording makes use of the temperature dependence of the $K_{u}$ and $H_{c}$ of recording media. In a hybrid recording scheme, as shown in Fig.2, writing is done at an elevated temperature (usually near the media's Curie Temperature $T_{c}$ ) where the $H_{c}$ of media reduces to the range of head writing field, data are archived at room temperature where very high $\mathrm{H}_{\mathrm{c}}$ ensures sufficient thermal stability and a high-sensitivity magnetic flux detector [for example, giant magnetoresistive (GMR) head or tunneling magnetoresistive (TMR) head] is utilized for data reading. Local heating of the media is commonly realized through a laser head or by laser through an optical fiber. These two heat sources and their different alignments, combined with writing magnetic field, determine the dimension and shape of the recording bit. At present, the media employed in hybrid recording are usually conventional longitudinal and perpendicular poly-crystalline HDD media or amorphous MO media, which also are schematically shown in Fig.2. Hybrid recording technology combines the advantages of Magneto-optical recording and magnetic recording so that high areal density as well as high data rate can be expected. Hybrid recording is a promising candidate for future magnetic recording with areal density beyond $1 \mathrm{Tbits} / \mathrm{in}^{2}$. 


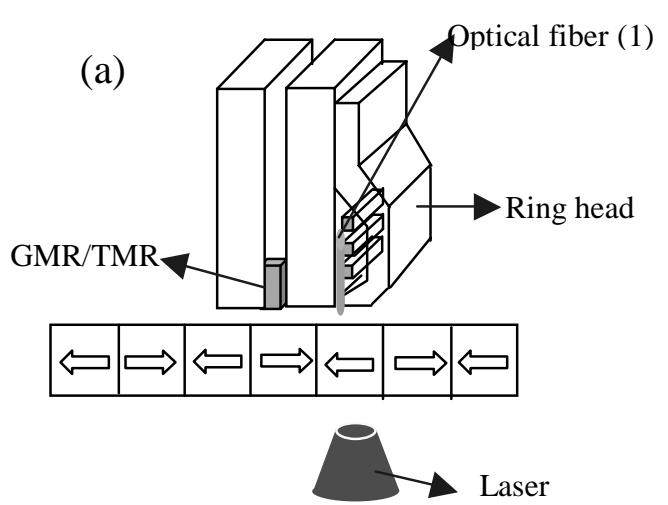

head (2)

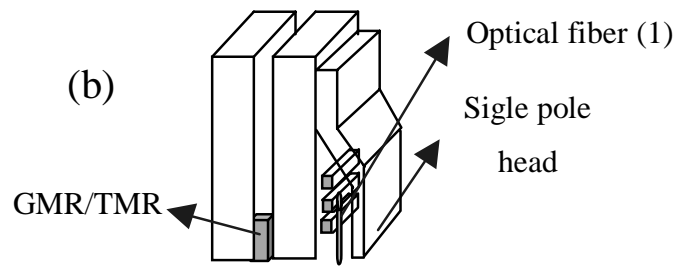

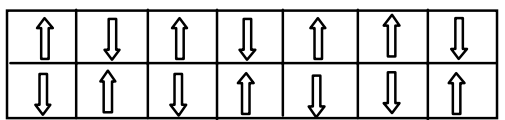

Laser

head (2)
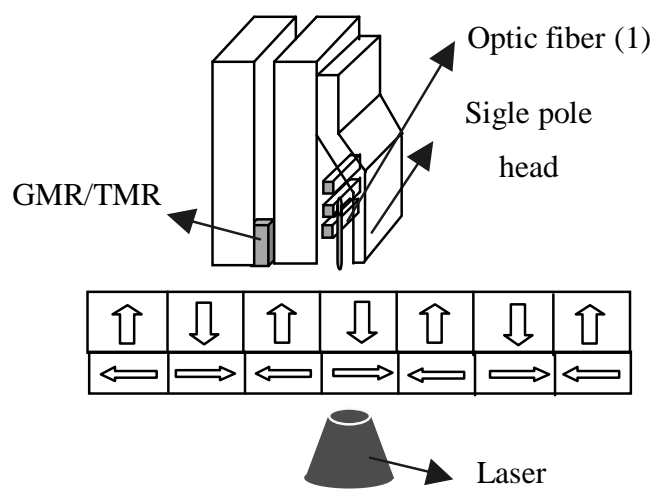

(c)

head (2)

Fig.2 Schematic configurations of hybrid recording using (a) longitudinal recording media (b) magneto-optical double-layer amorphous media and (c) perpendicular media with soft underlayer. [(1) and (2) refer to two different heat source alignments. ]

Extracting the challenges in the head design for hybrid recording, this report mainly focuses on the media design for hybrid recording and gives a review of the researches on the hybrid recording media in the world.

\section{Requirements for hybrid recording media}

Obviously, hybrid recording involves not only magnetic process but also thermal and optical process, which require its media have a combination of magnetic, thermal and tribological properties.

Basically, hybrid recording media should have high coercivity $\mathrm{H}_{\mathrm{c}}$, high magnetic crystalline anisotropy $K_{u}$ and adequate remanent magnetization $M_{r}$ at room temperature to ensure small transition length, enough thermal stability and read out signal. The coercivity of hybrid recording media at writing temperature $\mathrm{T}_{\mathrm{w}}$ should be 
no higher than 4500 Oe which is the highest writing field currently available for head materials. Since the writing temperature is near the media's Curie temperature $T_{c}$, hybrid recording requires $\mathrm{T}_{\mathrm{c}}$ not very high and be within the range of $150^{\circ} \mathrm{C}-230^{\circ} \mathrm{C}$ to keep low laser power consumption and avoid additional heating in the head-media interface. Reference [9] also suggests $\mathrm{M}_{\mathrm{r}} / \mathrm{H}_{\mathrm{c}}$ ratio at room temperature must be sufficiently low in order to avoid demagnetization and reduction of the decay time in saturated recording at the highest linear densities and to avoid transition widening. Additional requirements for hybrid recording media are the desirable temperature dependence of the magnetic properties and fast thermal response speed. Desirable temperature dependence of the magnetic properties refers to the ratio of $\mathrm{M}_{r}(T) / \mathrm{H}_{k}(T)$ $\left(\mathrm{H}_{\mathrm{k}}\right.$, anisotropy field and roughly twice the $\mathrm{H}_{\mathrm{c}}$ for polycrystalline magnetic media [1]) should be a constant in the range between room temperature and writing temperature to ensure the media's SNR improvement and areal density increase for polycrystalline media. At the same time, according to the Williams \& Comstock model for media transition length, transition length $a$ is proportional to $\left(\mathrm{M}_{\mathrm{r}} / \mathrm{H}_{\mathrm{c}}\right)^{1 / 2}{ }^{[10]}$. Therefore, the transition length $a$ would be temperature independent if the ratio of $\mathrm{M}_{\mathrm{r}}(\mathrm{T}) / \mathrm{H}_{\mathrm{c}}(\mathrm{T})$ keeps constant between room temperature and writing temperature. Furthermore, writing sharp transitions at a bit-aspect ratio larger than 1 requires a large temperature coefficient of the switching field at $\mathrm{T}_{\mathrm{w}}$ in hybrid recording scheme, which is given by $d H_{k} / d T^{[13]}$. The fast thermal response speed means the locally heated media can cool down rapidly enough to avoid magnetization erasure in writing process of neighboring bit and also means media can be heated rapidly to an elevated temperature where $\mathrm{H}_{\mathrm{c}}(\mathrm{T})$ drops momentarily well below the writing threshold of the head to realize high writing speed. T.W.McDaniel et al figured out that the media thermal response time for hybrid recording should be at or below the bit time of the disk, namely, $1 \mathrm{~ns}$ for the areal density of $1 \mathrm{~Tb} / \mathrm{in}^{2}$ with the bit aspect ratio (BAR) of $1: 1^{[11]}$. From the point of view of microstructure, like conventional recording media, the requirement for poly-crystalline hybrid recording media is to achieve exchange-decoupled grains, small enough grain size with narrow size distribution, preferred orientation, etc, to ensure good SNR and desired magnetic properties for high areal density recording. In sum, all these complex requirements for hybrid recording media imply much greater challenges in the design of hybrid recording media than in that of conventional longitudinal or perpendicular recording media.

\section{Review on the researches on hybrid recording media}

Currently, two types of hybrid recording media have been studied, one is the hard-disk polycrystalline media and the other is magneto-optical (MO) amorphous media. 


\subsection{Hard-disk polycrystalline media for hybrid recording}

S.R.Cumpson et al tried to use conventional CoCrPt-alloy magnetic recording media to hybrid recording system ${ }^{[12]}$. Although this kind of media has high $\mathrm{M}_{\mathrm{r}}$ at room temperature, its coercivity is not very high (usually less than $5000 \mathrm{Oe}$ ) so that it is writable directly by the head, the coercivity decreases much more slowly than MO media and the ratio of $\mathrm{M}_{\mathrm{r}}(\mathrm{T}) / \mathrm{H}_{\mathrm{c}}(\mathrm{T})$ is not constant between room temperature and writing temperature. These disadvantages limit the thin film's application in recording system of areal density beyond $100 \mathrm{~Gb} / \mathrm{in}^{2}$.

Binary alloy thin films with the face centered tetragonal (fct) phase, like CoPt and $\mathrm{FePt}$, show very high magnetic anisotropy $\mathrm{K}_{\mathrm{u}}$, making them attractive base materials for hybrid recording media ${ }^{[13,14]}$. Hybrid recording experiment on the multilayer $\mathrm{Co} / \mathrm{Pt}$ media with coercivity larger than $8 \mathrm{kOe}$ demonstrated high performance writability of this kind of media which can't be written at room temperature. This result suggests the great potential of fct phase thin films to be applied in hybrid recording system. However, the Curie temperatures $\left(\mathrm{T}_{\mathrm{c}}\right)$ of these films are relatively high (e.g., around $500^{\circ} \mathrm{C}$ for $\mathrm{FePt}$ ) and the writing temperatures are required close to $T_{c}$ to significantly decrease $H_{c}$ for enable writing. At such high temperatures, serious problems with the head-disk interface of the recording system such as lubricant degradation and large thermal stress can be expected. This problem was solved in $\mathrm{FeRh} / \mathrm{FePt}$ exchange spring films proposed by $\mathrm{J}-\mathrm{U}$ Thiele and his colleagues ${ }^{[15,16]}$. This novel thin film heterostructure consists of a first FePt layer with high anisotropy that is exchange-coupled to a second layer of FeRh or FeRhIr. FeRh transits from antiferromagnet to be ferromagnetic at an elevated temperature $\mathrm{T}_{\mathrm{AF}-\mathrm{FM}} \cdot \mathrm{T}_{\mathrm{AF}-\mathrm{FM}}$ is lower than $\mathrm{T}_{\mathrm{c}}$ and can be adjusted to about $200^{\circ} \mathrm{C}$ without significant reduction in $\mathrm{M}_{\mathrm{s}}$ by changing $\mathrm{Fe}-\mathrm{Rh}$ ratio or adding small amount of Ir. Through an exchange spring mechanism, at the write temperature $\mathrm{T}_{\mathrm{w}}$ for $\mathrm{FeRh} / \mathrm{FePt}$ bilayer $\left(\mathrm{T}_{\mathrm{AF}-\mathrm{FM}}<\mathrm{T}_{\mathrm{w}}<\mathrm{T}_{\mathrm{c}-\mathrm{FeRh}}\right), \mathrm{H}_{\mathrm{c}}$ is significantly lowered compared to single FePt layer. Therefore, $T_{w}$ is considerably lower than the $T_{c}$ of FePt single layer. Fig. 3 gives the temperature dependence of $H_{c}$ and $M_{s}$ of this bilayer structure. It shows that the $T_{w}$ is around $450 \mathrm{~K}$ although small temperature hysteresis exists. To further decrease this temperature hysteresis and simultaneously maintain the desired thermal and magnetic properties of this bilayer structure is a challenging task for its future practical application. Furthermore, adding Ni element in FePt equatomic thin film was believed another way to effectively reduce the writing temperature of FePt media ${ }^{[13]}$. The Curie temperature $\mathrm{T}_{\mathrm{c}}$ of $\mathrm{Fe}_{25} \mathrm{Ni}_{30} \mathrm{Pt}_{45}$ thin film is $490 \mathrm{~K}$, which is much lower than that of the binary FePt media $(770 \mathrm{~K})$, with a compromise of the magneto-crystalline anisotropy $\mathrm{K}_{\mathrm{u}}$ and saturation magnetization $\mathrm{M}_{\mathrm{s}}$. 


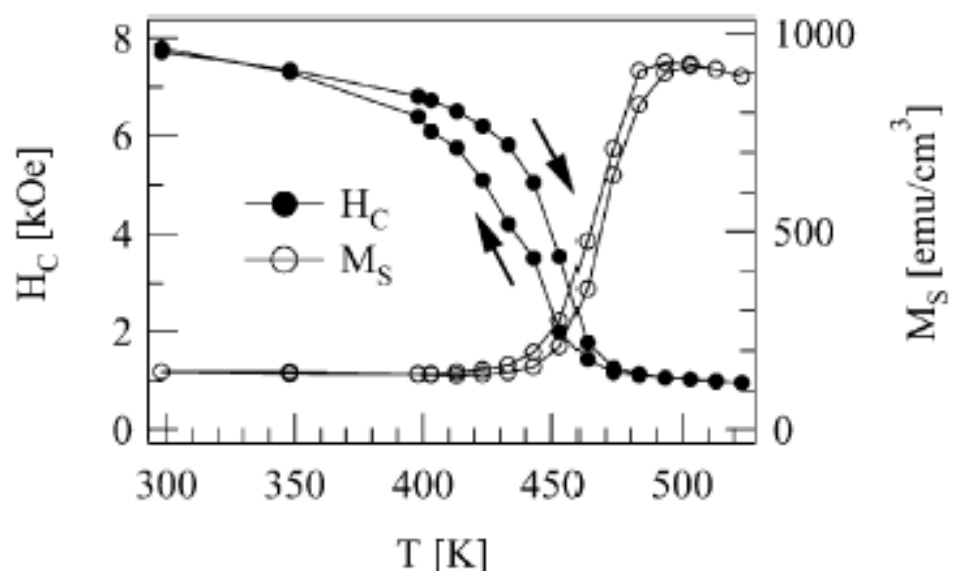

Fig.3 Temperature dependence of coercivity and saturation magnetization of a $25 \mathrm{~nm} \mathrm{FePt} / 125 \mathrm{~nm}$ Fe Rh Ir bilayer grown on a SiN-coated Si wafer. ${ }^{[16]}$

Synthetic ferrimagnetic media (SFM), also named antiferromagnetic media (AFM) were firstly used in longitudinal recording. Researchers in Fujitsu laboratory applied SFM media to hybrid recording recently ${ }^{[17]}$. Compared with conventional longitudinal recording media, SFM media with high $\mathrm{H}_{c}$ possesses better write-read characteristics.

\subsection{Magneto-optical amorphous media for hybrid recording}

Hybrid recording adopts thermomagnetic writing scheme like magneto-optical (MO) recording, so MO amorphous media are considered promising to be applied to hybrid recording ${ }^{[18-22]}$. Conventional MO medium, usually rare-earth-transition-metal (RE-TM) thin films, has large perpendicular anisotropy, therefore, very large perpendicular coercivity, which is very suitable for thermomagnetic writing. However, its $M_{r}$ and $M_{s}$ are so small that it is impossible to use GMR or TMR for the read-out signal detection. Therefore, to design RE-TM films with large $M_{r}$ or $M_{s}$ (at least $300 \mathrm{emu} / \mathrm{cm}^{3}$ ) is of significant importance in the research of MO amorphous media for hybrid recording.

H.Katayama et al tried single layer TbFeCo MO media in hybrid recording ${ }^{[21]}$. Although by adding AlN underlayer and adjust Fe:Co composition ratio the thermal and magnetic properties are greatly improved, the thin film $\mathrm{M}_{\mathrm{r}}$ is still very small. Study on CoTbAg single layer amorphous thin film shows that $M_{s}$ up to $310 \mathrm{emu} / \mathrm{cm}^{3}$, $\mathrm{M}_{\mathrm{r}}$ of $255 \mathrm{emu} / \mathrm{cm}^{3}$ and high perpendicular $\mathrm{H}_{\mathrm{c}}$ of $3100 \mathrm{Oe}$ were obtained in $\mathrm{Co}_{67.23} \mathrm{~Tb}_{30.52} \mathrm{Ag}_{2.25}$ film at room temperature ${ }^{[22]}$. Fig.4 gives the M-H loop and the temperature dependence of $\mathrm{H}_{\mathrm{c}}$ and $\mathrm{M}_{\mathrm{s}}$ of this film. These improved properties gave a clue of its application in hybrid recording. 

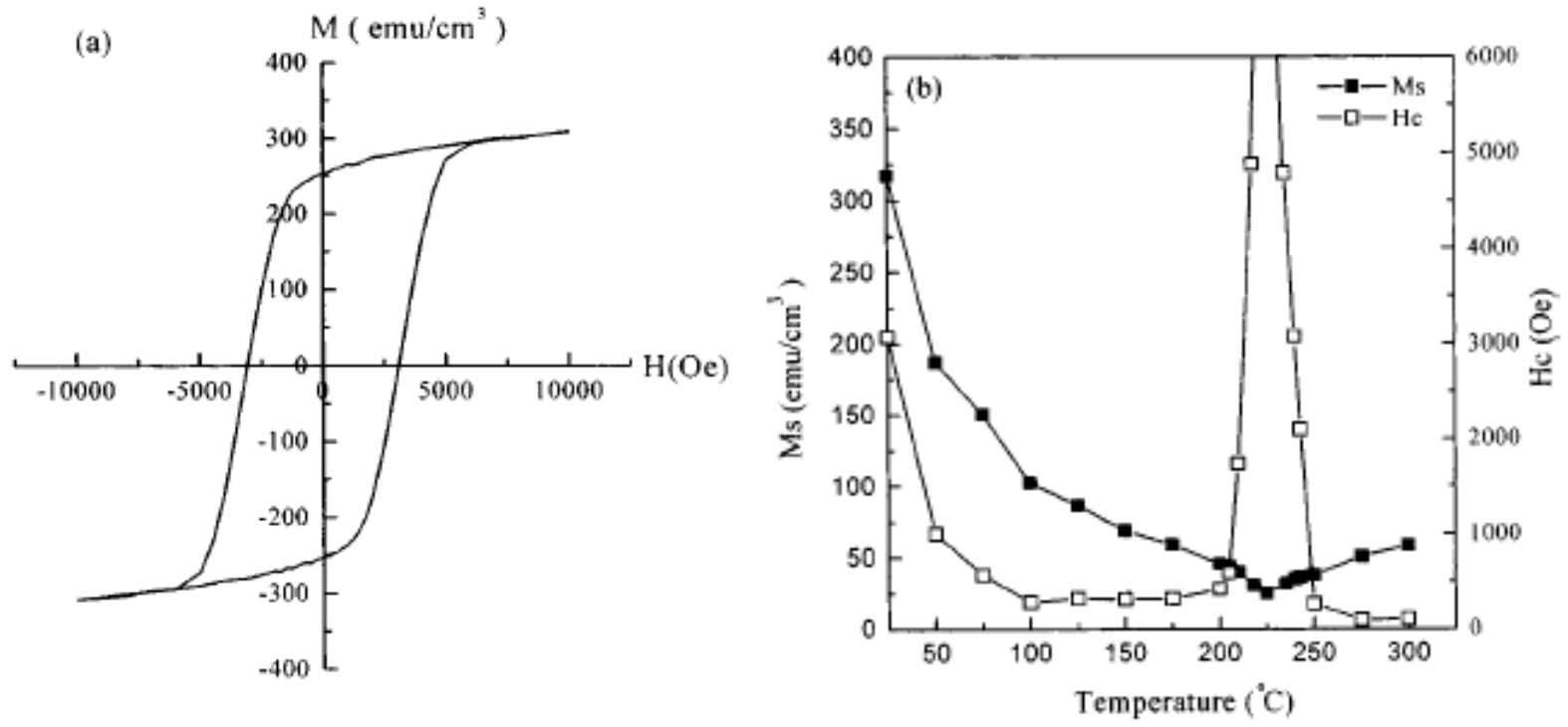

Fig.4 (a) the M-H loop and (b) the temperature dependence of $H_{c}$ and $M_{s}$ of the $\mathrm{Co}_{67.23} \mathrm{~Tb}_{30.52} \mathrm{Ag}_{2.25}$ single-layer amorphous film ${ }^{\text {[22] }}$

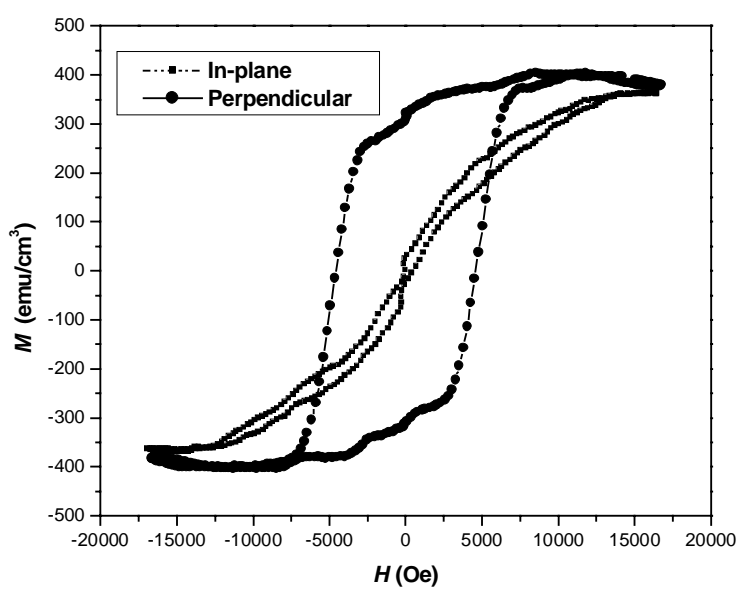

(a)

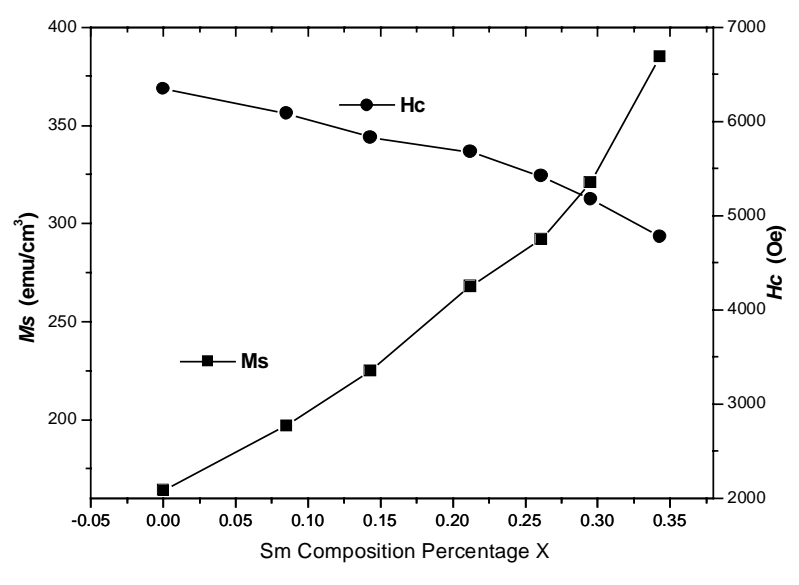

(b)

Fig. 5. (a) The in-plane and perpendicular M-H loops of a $120 \mathrm{~nm}\left(\mathrm{Sm}_{0.343} \mathrm{~Tb}_{0.657}\right)_{31} \mathrm{Co}_{69}$ film with a 180nm Cr underlayer.and (b) Composition dependence of saturation magnetization $\mathrm{M}_{\mathrm{s}}$ and coercivity $\mathrm{H}_{\mathrm{c}}$ of amorphous $\left(\mathrm{Sm}_{\mathrm{x}} \mathrm{Tb}_{1-\mathrm{x}}\right)_{31} \mathrm{Co}_{69}(120 \mathrm{~nm}) / \mathrm{Cr}(180 \mathrm{~nm})$ films ${ }^{[27]}$.

SmTbCo light-rare-earth-heavy-rare-earth-transition-metal (LRE-HRE-TM) thin film with $\mathrm{Cr}$ underlayer was developed in our lab ${ }^{[27]}$. With increasing Sm composition, it was found the $\mathrm{M}_{\mathrm{s}}$ increases without compromising a lot in $\mathrm{H}_{\mathrm{c}}$, and $\mathrm{M}_{\mathrm{s}}$ as high as $385 \mathrm{emu} / \mathrm{cm}^{3}$ and coercivity $\mathrm{H}_{\mathrm{c}}$ about $4.7 \mathrm{~K}$ Oe were obtained in the $\left(\mathrm{Sm}_{0.343} \mathrm{~Tb}_{0.657}\right)_{31} \mathrm{Co}_{69} / \mathrm{Cr}$ thin film, as shown in Fig.5. This result suggests that large 
$\mathrm{M}_{\mathrm{s}}$ can be obtained by adding LRE element into RE-TM amorphous MO media for hybrid recording. At present, we are working on depositing $\mathrm{SmTbCo/TbCo}$ and $\mathrm{SmDyCo} / \mathrm{TbFeCo}$ exchange-coupled amorphous media and better magnetic and thermal properties, which will result in satisfactory recording and readout characteristics, are anticipated.

\begin{tabular}{|l|}
\hline Protective layer \\
\hline Readout layer \\
\hline Memory Layer \\
\hline Protective layer \\
\hline Si Substrate \\
\hline
\end{tabular}

Fig.6 typical film structure of exchange-coupled double layer amorphous MO media for hybrid recording

Besides the single layer MO media, exchange-coupled double layer amorphous MO media has attracted much attention ${ }^{[23-25]}$. The typical film structure is shown in Fig.6. The readout layer and memory layer have different material composition and different magnetic properties. For example, the readout layer is made of TM-rich $\mathrm{TbFeCo}$ or $\mathrm{TbDyFeCo}$, and the memory layer is made of RE-rich TbFeCo. Magnetic property requirement for the readout layer is large $M_{s}$ or $M_{r}$ while for the memory layer is large $\mathrm{H}_{\mathrm{c}}$. Ms value of $370 \mathrm{emu} / \mathrm{cm}^{3}$ and $\mathrm{M}_{\mathrm{r}}$ value of $335 \mathrm{emu} / \mathrm{cm}^{3}$, which make flux detection possible, have been obtained in such kind of media ${ }^{[25]}$. In this double-layer structure, the readout layer is strongly exchange-coupled to the recording layer in order to copy out the recorded magnetic domains precisely. The Curie temperature of the readout layer is lower than that of the recording layer. In the thermo-magnetic writing process, magnetic domains are formed on the recording layer first and copied to the readout layer after the film temperature falls. Write-read experiments on this exchange-coupled double-layer MO media confirmed it is suitable for hybrid recording with an areal density of $60 \mathrm{Gbit} / \mathrm{in}^{2}{ }^{[24]}$.

The properties of above-mentioned media are summarized in Table 1.

\subsection{Thermal response speed of hybrid recording media}

In hybrid recording, the thermal response involves two processes, one is the heating process of the spot irradiated by a heating source (e.g. a laser beam) and the other is the subsequent cooling down after the heating source is removed, that is, the thermomagntic writing is completed. Thermal response speed determines the writing speed of hybrid recording and the stability of the written bit. For hybrid recording media, the thermomagnetic switching speed of the magnetic layer and the thermal design of the film and substrate play very important role in the thermal response speed improvement.

The ultrafast rewritable storage experiments on $\mathrm{GdFeCo}$ changed the conventional understanding that the thermomagnetic switching speed of MO media is much slower 
than that of the hard-disk media ${ }^{[20,26]}$. It is revealed that the complete magnetization reversal and rewritable response of GdFeCo realizes in a less than 1 picosecond period, which enables a very attractive application in hybrid recording scheme. In the thermal design of the hybrid recording media, computational simulation results suggest that employing heat-sinking layer in the media, which is widely used in MO media research, be an effective approach to shorten the thermal response time ${ }^{[11]}$. This kind of thermal design has been applied experimentally to the single-layer and exchange-coupled double layer amorphous MO media for hybrid recording and the heat-sinking layers are $\mathrm{AlN}$ and $\mathrm{Al}$ layer, respectively, although more intensive experimental investigation is needed on the effect of heat-sinking layer on the thermal response speed of hybrid recording media ${ }^{[21,24]}$.

Table 1 Properties of thin film media for hybrid recording

\begin{tabular}{|c|c|c|c|c|c|c|}
\hline \multicolumn{2}{|l|}{ Media } & $\mathrm{H}_{\mathrm{c}}(\mathrm{KOe})$ & $\begin{array}{l}\mathrm{M}_{\mathrm{s}}(\mathrm{emu} \\
\left./ \mathrm{cm}^{3}\right)\end{array}$ & $\begin{array}{c}\mathrm{M}_{\mathrm{r}}(\mathrm{emu} \\
\left./ \mathrm{cm}^{3}\right)\end{array}$ & $\mathrm{T}_{\mathrm{c}}(\mathrm{K})$ & $\begin{array}{l}\mathrm{dH}_{\mathrm{k}} / \mathrm{dT} \\
(\mathrm{Oe} / \mathrm{K}) \\
\end{array}$ \\
\hline \multicolumn{2}{|l|}{$(\mathrm{Co} / \mathrm{Pt})_{16}{ }^{[14]}$} & $>8$ & 447 & $\approx 400$ & ---- & $10-100$ \\
\hline \multicolumn{2}{|l|}{$\mathrm{FePt}{ }^{[13]}$} & 41 & 1125 & ---- & 770 & ---- \\
\hline \multicolumn{2}{|c|}{$\mathrm{Fe}_{25} \mathrm{Ni}_{30} \mathrm{Pt}_{45}[13]$} & 16 & 795 & $\begin{array}{ll}--- \\
\end{array}$ & 490 & 90 \\
\hline \multicolumn{2}{|c|}{$\mathrm{FePt} / \mathrm{FeRh}{ }^{[15,16]}$} & 8 & 100 & $\begin{array}{ll}--- \\
\end{array}$ & 450 & 500 \\
\hline \multicolumn{2}{|c|}{ CoTbAg ${ }^{[22]}$} & 3.1 & 310 & 255 & 473 & ---- \\
\hline \multicolumn{2}{|c|}{$\mathrm{C} / \mathrm{TbFeCo} / \mathrm{AlN}^{[21]}$} & $>20$ & 70 & ---- & 523 & ---- \\
\hline \multicolumn{2}{|c|}{$\mathrm{SmTbCo} / \mathrm{Cr}^{[27]}$} & 4.7 & 385 & ---- & 483 & ---- \\
\hline \multirow{2}{*}{$\begin{array}{l}\text { SiN/TM-rich-TbFeCo/RE } \\
\text {-rich-TbFeCo/SiN }\end{array}$} & TM rich & 0.95 & ---- & 113 & 473 & ---- \\
\hline & RE rich & 4.6 & 447 & $\approx 400$ & 543 & ---- \\
\hline \multirow{2}{*}{$\begin{array}{l}\mathrm{SiN} / \mathrm{Al} / \mathrm{SiN} / \mathrm{TbDyFeCo} / \\
\mathrm{TbFeCo} / \mathrm{SiN}^{[24]}\end{array}$} & TbDyFeCo & $<2$ & 260 & ---- & 513 & ---- \\
\hline & $\mathrm{TbFeCo}$ & $>10$ & $<100$ & ---- & 573 & ---- \\
\hline
\end{tabular}

(Note: $\mathrm{H}_{\mathrm{c}}$ is the coercivity at room temperature; $\mathrm{M}_{\mathrm{s}}$ saturation magnetization; $\mathrm{M}_{\mathrm{r}}$ remenant magnetization; $\mathrm{T}_{\mathrm{c}}$ Curie temperature; $\mathrm{dH}_{\mathrm{k}} / \mathrm{dT}$ temperature coefficient of the switching field at writing field.)

\section{Summary}

As a promising technology for the data storage with areal density beyond $1 \mathrm{~Tb} / \mathrm{in}^{2}$, hybrid recording has been attracting much attention and is studied intensively. Hybrid recording media is believed more complex than conventional longitudinal and perpendicular recording media for it should meet the requirements based on the considerations in magnetic, thermal and optical properties. This report gives a review 
on the researches on advanced hybrid recording media. Although they still are far away from the recording beyond $1 \mathrm{~Tb} / \mathrm{in}^{2}$, they are the first step to such a target.

\section{References:}

[1] S.H.Charap, P.Lu and Y.Ho: IEEE Trans. Magn., 33 (1997) 978

[2] D.Weller and A.Moser: IEEE Trans. Magn., 35 (1999) 4423

[3] D. Weller, A. Moser, L. Folks, M. E. Best, W. Lee, M. F. Toney, J.-U.Thiele, and M. F. Doerner, IEEE Trans. Magn. 36, (2000) 10

[4] K.Ohashi and Y.Yasue, IEEE Trans. Magn., 34 (1998) 1462

[5] T.E. Schlesinger, E. Black, W. Guo, L. Zhou, , A. Itagi, D.Stancil, J. A. Bain, Proceeding of Moris'2004 Mo-S-2

[6] K.Shimazaki, M.Yoshihiro, O.Ishizaki, S.Ohnuki, and N.Ohta, J.Magn.Soc.Jpn. 19, Suppl.S1(1995) 429

[7] H. Saga, H. Nemoto, H. Sukeda, and M. Takahashi, Jpn. J.Appl. Phys., vol. 38, (1999) 1839

[8] N.Ota, S.Matsunuma, T.Takeuchi, H.Daimon, N.Inaba, H.Awano, IEEE (2003) WA-02

[9] J. J. M. Ruigrok, R. Coehoorn, S. R. Cumpson, and H. W. Kesteren: J. Appl. Phys., 87 (2000) 5398

[10] M.L.Williams and R.L.Comstock, A.I.P Conf.Proc.,5 (1972) 738

[11] T.W.McDaniel and W.A.Challener, J.Magn.Soc.Jpn. 26, (2002) 316

[12] S. R. Cumpson, P. Hidding, and R. Coehoorn: IEEE Trans. Magn., 36 (2000) 2271

[13] J.-U. Thiele. K.R. Coffey., M. F. Toney, J. A. Hedstrom, and A. J. Kellock: J. Appl. Phys., 91 (2002) 6595.

[14] M.Alex, T.Valet, T.McDaniel. C.Brucker, J.Magn.Soc.Jpn.25, (2001) 328

[15] Jan-Ulrich Thiele, Stefan Maat, and Eric E. Fullerton: Appl. Phys. Lett., 82 (2003) 2859

[16] Jan-Ulrich Thiele, Stefan Maat, J. Lee Robertson, and Eric E. Fullerton: IEEE Trans. Magn., 40 (2004) 2537

[17] K. Matsumoto, J. Taguchi, A. Inomata, A. Ajan, W. Yamagishi, Proceeding of Moris'2004 Mo-S-4

[18] K.Nagata, Y.Kawakubo, D.Kato, T.Sugiyama, IEEE (2002) TU-P-09-01

[19] K.Kojima, S.Miyanishi, J.Sato, K.Takayama, H.Fuji, H.Katayama, K.Ohta, Trans.Magn.Soc.Jpn,2., (2002) 328

[20] N.Ota, M.Sekine, H.Awano, M.Bilderbeek, J.Hohfeld and T.Rasing, Jpn.J.Appl.Phys. 41 (2002) 1650

[21] Hiroyuki Katayama, Masaki Hamamoto, Jun-ichi Sato, Yoshiteru Murakami, and Kunio Kojima: IEEE Trans. Magn., 36 (2000) 195 
[22] C. T. Lie, P. C. Kuo, and C. L. Shen, J. Appl. Phys., 94 (2003) 2538

[23] H.Nemoto, H.Saga, Jpn.J.Appl.Phys., 38, Part 1, (1999) 1841

[24] H.Sukeda, H.Saga, H.Nemoto, Y.Itou, C.Haginoya, and T.Matsumoto, IEEE Trans. Magn., 37 (2001) 1234

[25] C.C.Lin, C.H.Lai, B. M. Chen, and H.P.Shieh, IEEE Trans. Magn., 37 (2001) 1399

[26] J.Hohlfeld, T.Gerrits, M.Bilderbeek, H.Awano, N.Ohta, J.Magn.Soc.Japan, 25, ( 2001) 202

[27] Z.Y.Li, Z.X.Huang, G.Q.Lin, F.Jin, Z.Li, Chin.Phys.Lett., 20 (2003) 909 